8-9-2013

\title{
Writing and Deleting Single Magnetic Skyrmions
}

\author{
Niklas Romming \\ University of Hamburg \\ Christian Hanneken \\ Matthias Menzel \\ Jessica E. Bickel \\ Cleveland State University, j.e.bickel@csuohio.edu \\ Boris Wolter
}

See next page for additional authors

Follow this and additional works at: https://engagedscholarship.csuohio.edu/sciphysics_facpub

Part of the Physics Commons

How does access to this work benefit you? Let us know!

\section{Publisher's Statement}

This is the author's version of the work. It is posted here by permission of the AAAS for personal use, not for redistribution. The definitive version was published in Science on 341 and August 9. 2013, DOI: $10.1126 /$ science. 1240573

\section{Repository Citation}

Romming, Niklas; Hanneken, Christian; Menzel, Matthias; Bickel, Jessica E.; Wolter, Boris; von Bergmann, Kirsten; Kubetzka, André; and Wiesendanger, Roland, "Writing and Deleting Single Magnetic Skyrmions" (2013). Physics Faculty Publications. 198.

https://engagedscholarship.csuohio.edu/sciphysics_facpub/198

This Article is brought to you for free and open access by the Physics Department at EngagedScholarship@CSU. It has been accepted for inclusion in Physics Faculty Publications by an authorized administrator of EngagedScholarship@CSU. For more information, please contact library.es@csuohio.edu. 


\section{Authors}

Niklas Romming, Christian Hanneken, Matthias Menzel, Jessica E. Bickel, Boris Wolter, Kirsten von

Bergmann, André Kubetzka, and Roland Wiesendanger 


\section{Writing and Deleting Single Magnetic Skyrmions}

Niklas Romming, Christian Hanneken, Matthias Menzel, Jessica E. Bickel, Boris Wolter, Kirsten von Bergmann, André Kubetzka, Roland Wiesendanger

Topologically nontrivial spin textures have recently been investigated for spintronic applications. Here, we report on an ultrathin magnetic film in which individual skyrmions can be written and deleted in a controlled fashion with local spin-polarized currents from a scanning tunneling microscope. An external magnetic field is used to tune the energy landscape, and the temperature is adjusted to prevent thermally activated switching between topologically distinct states.

Switching rate and direction can then be controlled by the parameters used for current injection. The creation and annihilation of individual magnetic skyrmions demonstrates the potential for topological charge in future information-storage concepts.

$\mathrm{M}$ agnetic skyrmions $(I)$ are topologically stable, particle like spin configurations that carry a characteristic topological charge $S$, which is essentially a measure of the magnetization curvature. For a surface area $A$,

$S$ is defined as $S=\frac{1}{4 \pi} \int_{A} \mathbf{n} \cdot\left(\frac{\partial \mathrm{n}}{\partial x} \times \frac{\partial \mathrm{n}}{\partial y}\right) d x d y$, where

$\mathbf{n}$ is the normalized magnetization vector and $x$ and $y$ are the spatial coordinates. A single skyrmion carries a quantized charge of $S+1$; for an antiskyrmion, the charge is $S \quad 1$. In contrast, a spin spiral and the ferromagnetic (FM) 
state are topologically trivial with $S \quad 0$. Transitions between such topologically distinct states are forbidden within a continuous descrip tion of $\mathbf{n}$, but in a real system with magnetic moments on an atomic lattice, strict topological protection does not exist. Instead, the states are separated by a finite energy barrier. Magnetic skyrmions typically arrange in two dimensional (2D) lattices (2 4). An important prerequisite for their formation is broken inversion symmetry, which is fulfilled not only for samples with chiral crystal structure (4 9), but also for magnetic film systems, in which the top and bottom interfaces are different $(10$ 12). In recent years, magnetic skyrmions have been observed in a number of systems with broken crystal inversion symmetry ranging from metallic and semiconducting (4 7) to insulating $(8,9)$. Each of these systems dis plays a spin spiral phase in zero field (Fig. 1A), resulting from a competition of magnetic ex change and Dzyaloshinskii Moriya interaction, and can be driven into a hexagonal skyrmion lat tice phase (Fig. 1B) by the application of an ex ternal magnetic field $B$. In contrast to the spin spiral state, which is magnetically compensated, the hexagonal skyrmion lattice exhibits a net mag netization and is, therefore, favored by the Zeeman energy. By further increasing the magnetic field, the parallel alignment of the magnetic moments becomes energetically more and more favorable, until the skyrmion phase is eventually saturated to the FM phase (Fig. 1C) (5). Both of these phase transitions are accompanied by a change of topo logical charge. In thin films, the skyrmion lattice phase space increases as the thickness of the sample is decreased (7). In such 2D systems, the diameter of the skyrmions can exceed the film thickness $(5,7)$, which may be favorable for con trolled skyrmion manipulation by surface tech niques. At the ultimate limit, a single atomic layer of $\mathrm{Fe}$ on $\operatorname{Ir}(111)$ exhibits a skyrmion lattice, even in the absence of an external field. This skyrmion lattice has square symmetry and a period of only $1 \mathrm{~nm}$ (12); however, driving it into a different topological state has been challenging.

The manipulation of skyrmion lattices may require substantially smaller current densities com pared with the manipulation of domain walls in conventional ferromagnetic systems, which makes skyrmions a promising candidate for spintronic applications (13 18). However, even though sin gle skyrmions were observed experimentally (5), and the creation of skyrmions by radial currents was studied theoretically (19), the manipula tion or creation of individual skyrmions has presented difficulties. To design an ultrathin film system exhibiting skyrmions that can be manip ulated with a local probe, we cover the Fe layer on $\operatorname{Ir}(111)$ with an additional atomic layer of Pd (20). We thereby modify the top interface and, thus, the magnetic interactions within the film. As Pd is known to be easily polarized by adjacent magnetic moments (21), we expect the resulting PdFe bilayer to behave as a single mag netic entity.

Figure 1 shows the magnetic field dependence of the PdFe bilayer on $\operatorname{Ir}(111)$ at a temperature $T$ of $8 \mathrm{~K}$. In zero field (Fig. 1D), the spin polarized

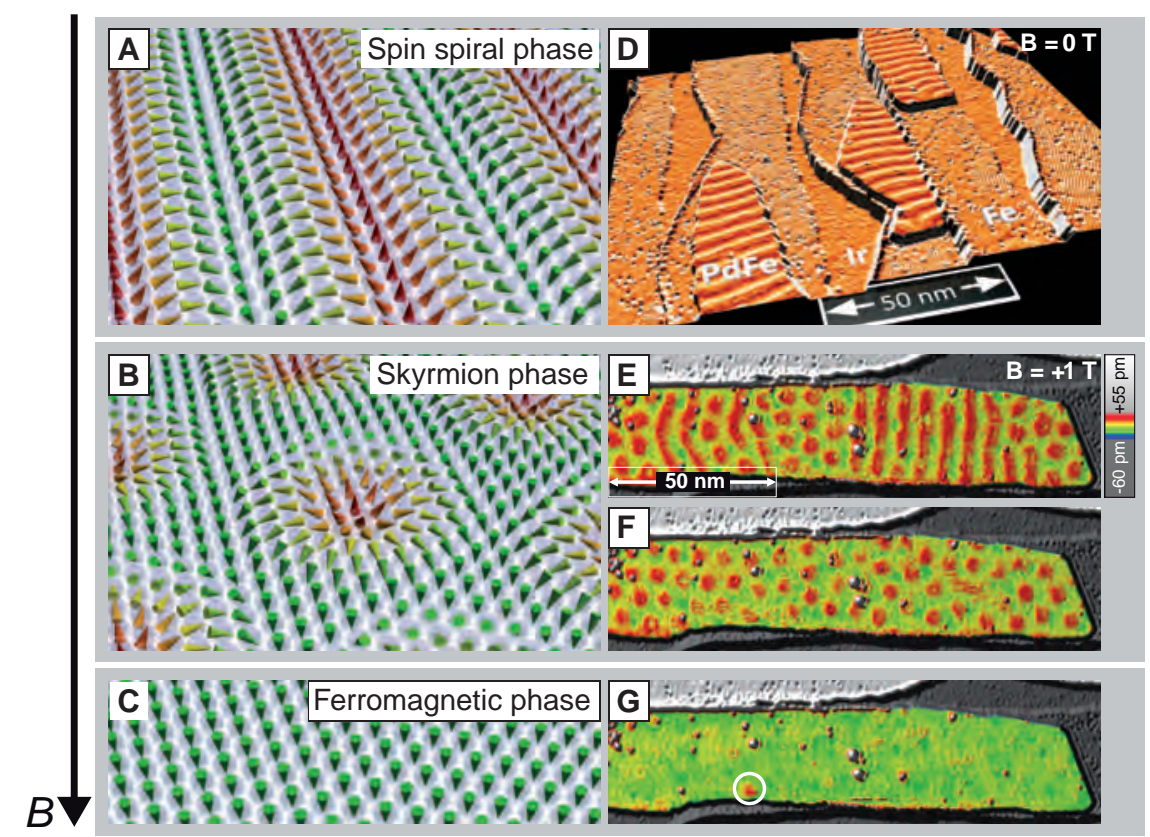

Fig. 1. Magnetic field dependence of the PdFe bilayer on the $\operatorname{Ir}(111)$ surface at $T=8 \mathrm{~K}$. (A to C) Perspective sketches of the magnetic phases. (D) Overview SP-STM image, perspective view of constant-current image colorized with its derivative. (E to $\mathbf{G}$ ) PdFe bilayer at different magnetic fields ( $U=+50 \mathrm{mV}, I=0.2 \mathrm{nA}$, magnetically out-of-plane sensitive tip). (E) Coexistence of spin spiral and skyrmion phase. (F) Pure skyrmion phase. (G) Ferromagnetic phase. A remaining skyrmion is marked by the white circle.

scanning tunneling microscopy (SP STM) image (20) reveals a spin spiral ground state with a pe riod of 6 to $7 \mathrm{~nm}$. When the magnetic field is increased to $B+1 \mathrm{~T}$ (Fig. 1E), skyrmions can be observed coexisting with remaining areas of spiral ordering. Even higher values of $B$ lead to a pure hexagonal skyrmion lattice (Fig. 1F) and, eventually, to saturation of the film to a FM phase (Fig. 1G); here only a few single skyrmions, pinned at atomic defects, remain at $B+2 \mathrm{~T}$. This field dependent behavior is similar to previously studied systems (5), and the transitions are reversible at $T \quad 8 \mathrm{~K}$ : Removing the field leads to qualitatively the same magnetic structure as in Fig. 1D, with small variations in the details of the spiral posi tion and direction.

Figure 2A depicts a comparable sample at $B \quad+1 \mathrm{~T}$, this time measured at $T 4.2 \mathrm{~K}$. Whereas the trend of skyrmion formation at the cost of the spiral phase is similar, fewer skyrmions are observed compared with the measurement at $T \quad 8 \mathrm{~K}$ (see Fig. 1E). This may be partly due to a small shift with temperature of the critical mag netic field needed for the phase transition $(4,5,7)$ or to small differences in the local environment. However, the main reason is reduced thermal en ergy, which prevents the system from reaching energetically lower states. A change of $B$ at $T$ $4.2 \mathrm{~K}$ may not necessarily lead to the lowest energy state; instead, a metastable state may be preserved. We demonstrated a transition from a metastable to a lower energy state by depositing energy into the system by tunneling with higher energy electrons: After scanning the surface area at an increased bias voltage of $U+1 \mathrm{~V}$, the spin spiral has locally transformed into skyrmions (Fig. 2B). A higher degree of control can be achieved by injecting higher energy electrons lo cally, as in Fig. 2, C and D, where skyrmions are imprinted into the ferromagnetic phase; by volt age sweeps with the STM tip held stationary, skyrmions can be created one by one below the tip or in close vicinity (Fig. 2E). Apparently, atomic defects act as preferred nucleation and pinning sites (see also fig. S2). In such a writing process, a topological charge $S$ is created. The field dependent potential can be sketched as in Fig. $2 \mathrm{~F}$, where $B_{0}$ is the field at which the two states are energetically degenerate. For $B<B_{0}$, the energetically lower state is the skyrmion $(S$ $1)$; for $B>B_{0}$, it is the FM state $\left(\begin{array}{ll}S & 0\end{array}\right)$. When we start from the FM state and lower the external field to $B<B_{0}$ (as in Fig. 2C), the system can be transferred to the skyrmion state only if the remaining energy barrier is over come, either by thermal fluctuations or if en ergy is supplied by other means (e.g., by the tunneling electrons).

For magnetic field values close to $B_{0}$, skyrmions can be created and annihilated re versibly. In this way, topological charge can be used to store information, as demonstrated in Fig. 3: By locally injecting electrons, we can gen erate any desired skyrmion configuration for the four pinning sites within the area. In the series of 
difference SP STM images in Fig. 3, B to E, the skyrmions are annihilated one by one until no skyrmion is present (in Fig. 3F); skyrmions are then created in a different sequence until the start ing configuration is reached again (Fig. 3, G to J). The writing and deleting was done between the
Fig. 2. Manipulation of the magnetic states of the PdFe bilayer at $T=4.2 \mathrm{~K}$. $(A$ and B) SP-STM images at $B=+1 \mathrm{~T}(U=+100 \mathrm{mV}$, $I=0.5 \mathrm{nA}$, magnetically inplane sensitive tip). Whereas (A) shows the sample in its initial magnetic state after sweeping the field up from $B=0 \mathrm{~T}$ to $+1 \mathrm{~T}$, in (B) the spin spiral has locally transformed into skyrmions after supplying energy by a highervoltage STM scan with $U=$ $+1 \mathrm{~V}, I=0.5 \mathrm{nA}$. (C) SPSTM image of the initial state at $B=+1.8 \mathrm{~T}$ after sweeping the magnetic field down from $+3 \mathrm{~T}$. Four skyrmions are marked by circles $(U=+100 \mathrm{mV}, I=$ $1 \mathrm{nA}$, magnetically out-ofplane sensitive tip). (D) Successive population of the island with skyrmions by injecting higher-energy electrons through local voltage sweeps (20) (fig. S2). (E) Concept of skyrmion manipulation with local currents from an STM tip. (F) Sketch of the field-dependent potential for a skyrmion (Sk; $S=1$ ) and the FM state
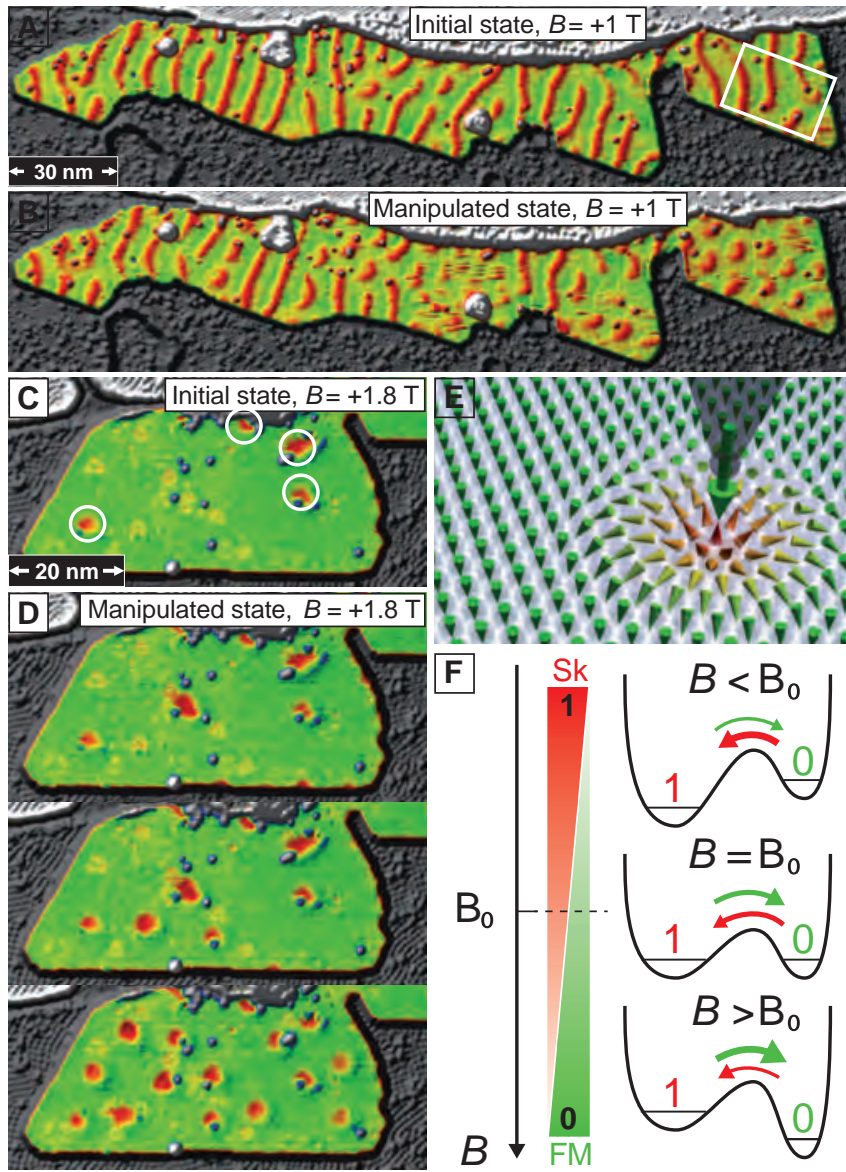

images by local voltage sweeps. This series dem onstrates that the skyrmions can be addressed individually and independently, even in close prox imity to one another.

Whereas controlled skyrmion creation and annihilation is demonstrated, the intermediate magnetization states during the switching process cannot be imaged directly because of the limited time resolution in our experiment. We consider the following mechanisms that may contribute to switching $(22,23)$ : (i) thermal noise, (ii) a local temperature increase caused by the injected pow er (Joule heating), (iii) nonthermal excitations from the injected electrons, and (iv) spin transfer torque (STT). The latter depends on the spin polarization of the tunnel current and its direction. To discriminate between these different contribu tions, we performed measurements as a function of bias voltage $U$, tunnel current $I$, and applied mag netic field $B(23,24)$ : With the tip held stationary above atomic pinning sites, we recorded the time evolution of the system (20). The observed mag netic telegraph noise (see insets in Fig. 4) oc curs because of repetitive switching between a skyrmion $\left(\begin{array}{ll}S & 1\end{array}\right)$ and the FM state $\left(\begin{array}{ll}S & 0\end{array}\right)$. From each measurement, typically consisting of 1000 switching events, the switching rate $f$ and the probability to observe a skyrmion $P$ can be ex tracted (22). We find that the switching process is very sensitive to the energy of the tunneling electrons $e U$ (where e is the elementary charge) (Fig. 4A): At $U \quad 300 \mathrm{mV}$, switching occurs, on average, once every $15 \mathrm{~s}$ at $I \quad 300 \mathrm{nA}$. Toward lower bias voltages, switching becomes increasing ly rare, which facilitates nonperturbing imaging. For higher voltages, the rate increases rapidly, allowing efficient skyrmion manipulation. In con trast, the current dependence at fixed bias voltage (Fig. 4B) is much weaker. The switching rate de pends linearly on $I$ within the investigated current

$(S=0) . B_{0}$ is the field where the two states are energetically degenerate.

Fig. 3. Creation and annihilation of single skymions. (A) Constantcurrent image of a sample region with four defects (see box in Fig. 2A), each hosting a skyrmion marked by a circle containing $\sim 270$ surface atoms $(U=$ $+250 \mathrm{mV}, I=1 \mathrm{nA}, B=$ $+3.25 \mathrm{~T}, T=4.2 \mathrm{~K}$, magnetically in-plane sensitive tip). (B to E) Sequence of difference SP-STM images [with respect to $(\mathrm{F})$ ] showing the selective erasing of all four skyrmions using local voltage sweeps (feedback loop switched off while bias voltage was increased to $+750 \mathrm{mV}$ ).

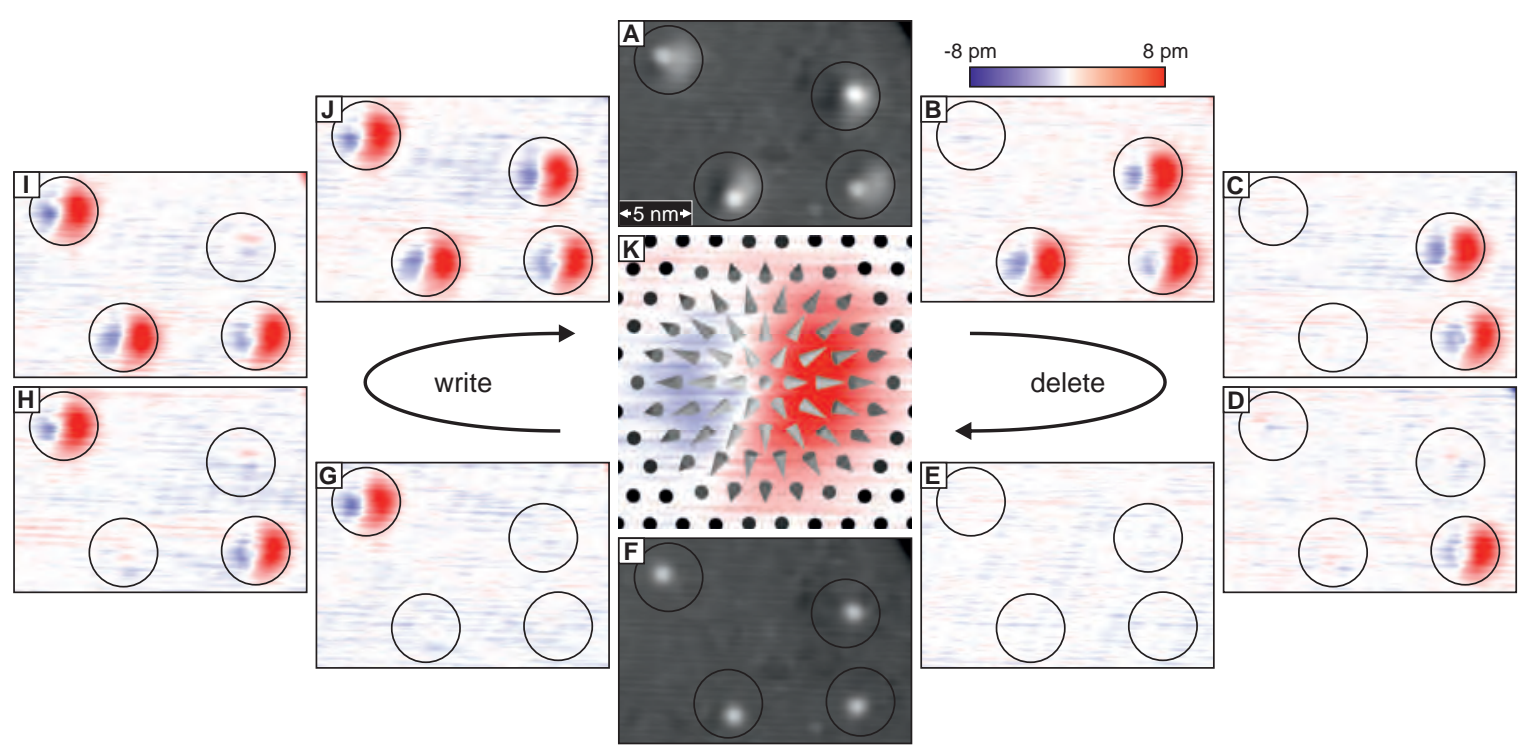

out skyrmions (constant-current image) and (G to ]) their successive rewriting (difference images). (K) Schematic spin configuration with distances twice the atomic lattice, superimposed on the experimental data: The asymmetric appearance of the skyrmions results from a canted SP-STM tip magnetization in this experiment. 

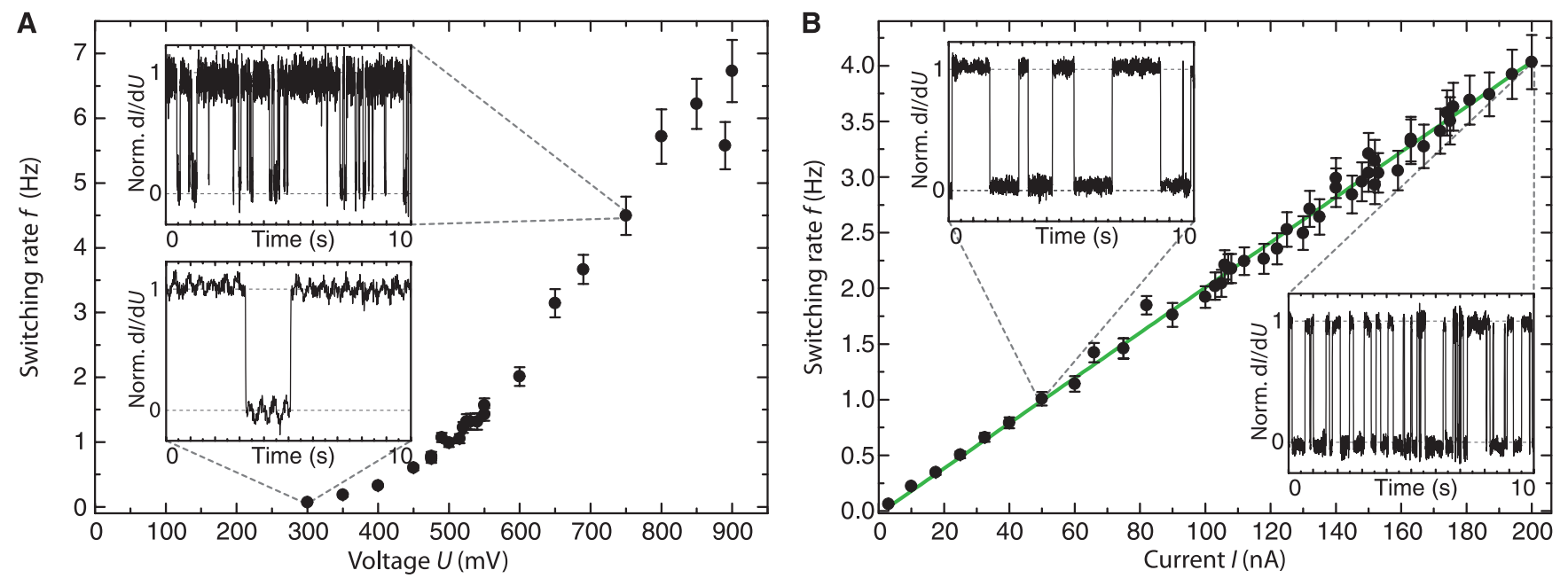

Fig. 4. Voltage, current, and magnetic field characteristics of skyrmion creation and annihilation at $\boldsymbol{T}=\mathbf{4 . 2} \mathrm{K}$. All data points are derived from magnetic telegraph noise (20), measured on top of atomic pinning sites with magnetically out-of-plane sensitive SP-STM tips. (A) Measured switching rates $f$ depend sensitively on $U(I=300 \mathrm{nA}, B=2.7 \mathrm{~T})$. (B) Switching rate increases linearly with tunnel current $I(U=650 \mathrm{mV}, B=2.9 \mathrm{~T})$. (C) Skyrmion probability decreases with increasing $B$ field. About $100 \mathrm{mT}$ are needed to compensate for different current directions at $U=+600$ and $-600 \mathrm{mV}$ (data points and fitted hyperbolic tangent functions, $I=100 \mathrm{nA}$ ). Norm., normalized; error bars, SD.

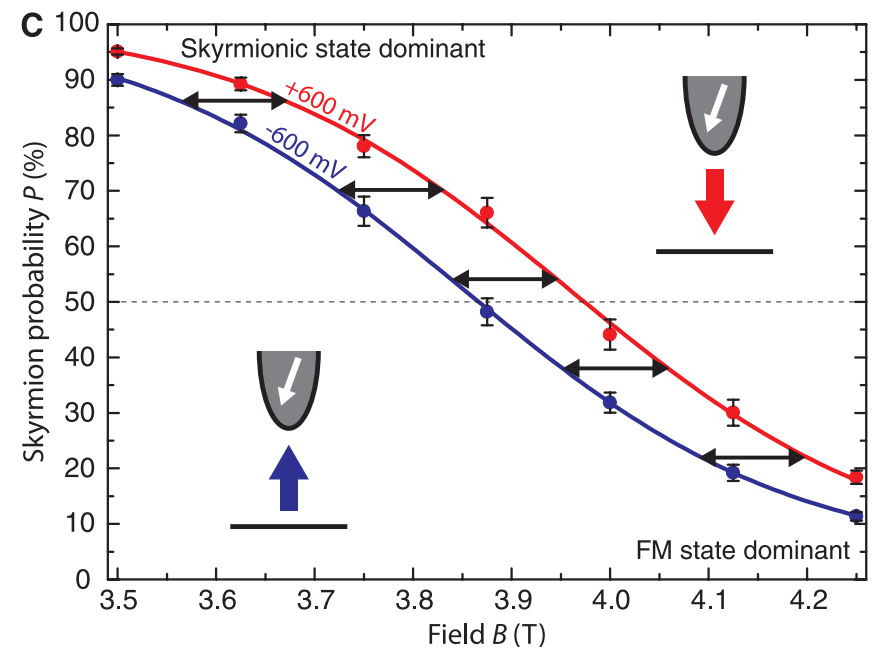

range, which means that, on average, the number of electrons needed for switching, $I /(f \cdot e)$, is con stant, independent of $I$. We found that, in the limit of low bias and low current, the switching rate approaches zero, which shows that thermally activated switching (i) does not play a role at $T \quad 4.2 \mathrm{~K}$. The injected power $I U$ is also not a decisive quantity for the switching process. At constant power, the switching rates still depend critically on $U$. Thus, local thermal heating (ii) can be ruled out as a driving mechanism. Instead, the energy of the injected electrons $|e U|$ (iii) ap pears to be the dominant factor determining the switching rate. Figure $4 \mathrm{C}$ shows the magnetic field dependent skyrmion probability $P$ for the two current directions, while $|e U|$ and $|I|$ are fixed. As expected, $P$ decreases with increasing $B$ (Fig. 2 F). More importantly, the data points show a uniform shift of $\Delta B \approx 100 \mathrm{mT}$ upon current re versal. Because all other parameters are fixed, this indicates that $\Delta B$ results from a difference of the STT (iv) at the chosen bias voltages (23). Along with the external field $B$, the STT is, thus, a means to control the directionality of the switch ing. Whereas the switching trajectory within the energy landscape and the coupling of the spin polarized tunnel current to the magnetic states re main to be identified for the optimization of the switching process, our work demonstrates the feasibility of using spin polarized tunnel cur rents for the controlled manipulation of individ ual skyrmions.

\section{References and Notes}

1. T. H. R. Skyrme, Nucl. Phys. 31, 556569 (1962).

2. A. Bogdanov, A. Hubert, J. Magn. Magn. Mater. 138, 255269 (1994)

3. U. K. Rößler, A. N. Bogdanov, C. Pfleiderer, Nature 442, 797801 (2006).

4. S. Mühlbauer et al., Science 323, 915919 (2009).

5. X. Z. Yu et al., Nature 465, 901904 (2010).

6. W. Münzer et al., Phys. Rev. B 81, 041203 (2010).

7. X. Z. Yu et al., Nat. Mater. 10, 106109 (2011).

8. T. Adams et al., Phys. Rev. Lett. 108, 237204 (2012).

9. S. Seki, X. Z. Yu, S. Ishiwata, Y. Tokura, Science 336 , 198201 (2012)

10. M. Bode et al., Nature 447, 190193 (2007).

11. P. Ferriani et al., Phys. Rev. Lett. 101, 027201 (2008).

12. S. Heinze et al., Nat. Phys. 7, 713718 (2011).

13. F. Jonietz et al., Science 330, 16481651 (2010).

14. N. S. Kiselev, A. N. Bogdanov, R. Schäfer, U. K. Rößler, J. Phys. D Appl. Phys. 44, 392001 (2011).

15. J. Zang, M. Mostovoy, J. H. Han, N. Nagaosa, Phys. Rev. Lett. 107, 136804 (2011).

16. T. Schulz et al., Nat. Phys. 8, 301304 (2012).
17. X. Z. Yu et al., Nat. Commun. 3, 988 (2012).

18. A. Fert, V. Cros, J. Sampaio, Nat. Nanotechnol. 8, 152156 (2013).

19. Y. Tchoe, ]. H. Han, Phys. Rev. B 85, 174416 (2012).

20. Materials and methods are available as supplementary materials on Science Online.

21. W. Weber, D. A. Wesner, G. Güntherodt, U. Linke, Phys. Rev. Lett. 66, 942945 (1991).

22. S. Krause, L. Berbil Bautista, G. Herzog, M. Bode, R. Wiesendanger, Science 317, 15371540 (2007).

23. A. A. Khajetoorians et al., Science 339, 5559 (2013).

24. S. Loth, S. Baumann, C. P. Lutz, D. M. Eigler, A. J. Heinrich, Science 335, 196199 (2012).

Acknowledgments: We thank S. Krause, A. A. Khajetoorians, A. Sonntag, S. Heinze, B. Dupé, and Ch. Hübner for discussions. We acknowledge financial support from the Deutsche Forschungsgemeinschaft via grants SFB668 and GrK 1286, the European Union via the European Research Council Advanced Grant FURORE, and the Hamburg Cluster of Excellence NANOSPINTRONICS.

Post-print standardized by MSL Academic Endeavors, the imprint of the Michael Schwartz Library at Cleveland State University, 2017. 